\title{
4. Two Autopsy Cases of Primary Duralsinus Thrombosis with Intracerebral Hemorrhage
}

\author{
T. Aizawa, Y. Goto, G. Araki, S. Hirai, K. Mori, \\ K. Murakami and N. Sato \\ Department of Internal Medicine, School of Medicine, Keio University \\ T. YAMAMURA \\ Department of Pathology, School of Medicine, Keio University
}

Two cases with primary dural sinus thrombosis, a rare form thrombosis, without infective focus, were presented.

\section{Case I 27 year old male}

This case admitted to Keio University Hospital with the X-Ray diaggnosis of mediastinal tumor in December 1961. During the admission, a histological diagnosis of lymphosarcoma was made by probe puncture. Hemograms and bone marrow smears revealed a large number of immature lymphocytes. Treatment with antineoplastic agents and $\mathrm{X}$-irradiation was started under a clinical diagnosis of leucosarcoma. On May 19, 1962, the the patient developed right hemiparesis in association with headache, nausea, vomiting, and later became quadriplegic. Papillar edema was noted in fundi and a spinal tap revealed high C.S.F. pressure. He fell into coma and died on May 23, 1962. Autopsy study revealed an acute myelogenous leucemia, and venous hemorrhages in the bilateral frontal and parietal lobes due to a superior sagittal sinus thrombosis.

\section{Case II 22 year old male}

This man was hospitalized in March, 1963, with the diagnosis of reticulosarcoma in the left thigh. After his condition has been proved to be inoperable, the treatment resorted to antineoplastic agent and X-irradiation. Severe pain in the bilateral legs with paresis, and sphincter disturbances resulted from a lumber spine metastasis in July. Headache, nausea, vomiting, dysphagia, diplopia, and nuchal rigidity were gradually added to his clinical picture, but ocular fundi was continuously normal. From the end of September, he took a downhill course and died on October 2. Autopsy findings included a reticulosarcoma of the left thigh with its metastosis, and hemorrhages in the left frontal, occipital, and bilateral temporal lobes due to multiple thrombosis in superior sagittal and lateral sinuses.

In both cases, it was proved by autopsy that dural sinus thrombosis developed as a complication of malignant tumor, namely leucemia in the 
first and reticulosarcoma in the second case. In the second case, motor weakness was masked by those signs of vertebral metastasis. Neither hemoconcentration or thrombocytosis was demonstrated in our cases. Furthermore, convulsive seizure, a common symptome of this disease, was not observed in these two cases.

\title{
5. Case Report :-Cerbral Hemorhage occured Immediately after Revascularization in a Patient with Complete Occulusion of Internal Carotid Artery
}

\author{
H. Imanaga, H. Nagai, K. Sakurai, M. Hayashi, M. Furuse, \\ K. Okamura, A. Shintani and T. Kobayashi \\ 2nd Department of Surgery, School of Medicine, Nagoya University
}

We present a case of cerebral hemorrhage occured immediately after carotid artery surgery in a patient with complete occulusion of right internal carotid artery. This is a report of a consideration upon the surgical treatment.

Case-A 43 year old male with complaints of left hemiplegia, dissturbances of consciousness, dysarthria and headache in several hours after apopletic attack.

On admission to the hospital, EEG findings revealed suppression in all areas, arteriogram showed complete occulasion at the bifurcation of right internal carotid artery and blood pressure was 170/110.

Thrombectomy and endarterectomy were performed eighteen days after attack; atheromatous plaque was found in the vessel wall. Backbleeding was assured.

Postoperatsvely rised blood pressure to 280 and appeared bradycardia, apnoea and right mydriasis. Echo-encephalogram demonstrated the shift of mid-line echo to the left.

Craniotomy was performed under the diagnosis of massive hemorhage in the infarcted tissue. We recognised diffuse hemorrhage to be widesperead in right hemisphere, cerebral parenchym to be softened and arterial vessel walls to be weak. The patient died 2 hours after ligation of right common carotid artery.

De Bakey, Wylie et al. recently reported some cases seized by cerebral hemorrhage following revasculartzation of stenosis or occulusion in the 